\title{
Sulfur and azobenzenes, a profitable liaison: straightforward synthesis of photoswitchable thioglycosides with tunable properties
}

\author{
Jonathan Berry ${ }^{\mathrm{a}}$, Thisbe K. Lindhorst ${ }^{\mathrm{a} *}$ and Guillaume Despras ${ }^{\mathrm{a}, \mathrm{b} *}$
}

[a] Otto Diels Institute of Organic Chemistry, Christiana Albertina University of Kiel, Otto-Hahn-Platz 3/4, 24118 Kiel, Germany.

[b] Present affiliation : Laboratoire des IMRCP, Université de Toulouse, CNRS UMR 5623, Université Paul Sabatier, 118 route de Narbonne, 31062 Toulouse Cedex 9, France. Email : despras@chimie.upstlse.fr

Keywords: azobenzene - glycoconjugates - thioarylation - photoswitching - visible light

\begin{abstract}
Azobenzene photoswitches are valuable tools for controlling properties of molecular systems with light. In particular, we have been investigating azobenzene glycoconjugates to probe carbohydrateprotein interactions and also to design photoresponsive glycomacrocycles with modulable chiroptical and physicochemical properties. To date, direct conjugation of glycosides to azobenzenes was performed by reactions providing target compounds in limited yields. We therefore sought for a more effective and reliable coupling method. In this paper, we report on a straightforward thioarylation of azobenzene derivatives with glycosyl thiols as well as other thiol substrates, increasing the scope of azobenzene conjugation. Importantly, red-shifted azoswitches that can be addressed with visible light were easily functionalized and challenging unsymmetrical conjugates were prepared in good yields via sequential or one pot procedures. In addition, we took advantage of the presence of the sulfide bridge to tune both photochromic and thermal relaxation properties of the core azobenzene via sulfur oxidation. Based on our photochemical investigations, we finally demonstrated orthogonal three-state photoswitching in mixtures containing two distinct azobenzene thioglycosides.
\end{abstract}

\section{Introduction}

Controlling properties of biomolecules in a reversible manner is a captivating task that holds formidable potential to study complex processes in living organisms. Light is an excellent external stimulus in such application because it offers a high degree of spatiotemporal resolution, can be precisely regulated, is often non-destructive in the visible range. Thus, chemists have been developing molecules that can be switched between two forms by means of light irradiation: photoswitches. ${ }^{1}$ Among those, azobenzene has arisen as one of the largest and most studied class of photochromic compounds. Indeed, it exhibits intense UV-visible absorption properties that can be finely tuned by substitution of the phenyl rings, and especially undergoes remarkable spatial modification upon trans/cis isomerisation about the azo bond. ${ }^{2}$

The combination of carbohydrates with azobenzene to form azobenzene glycoconjugates (ABGs) has emerged as a promising strategy to modulate macroscopic properties of carbohydrates in a spatiotemporally controlled manner. ${ }^{3}$ For instance, ABGs have found applications in material sciences as photoswitchable hydrogelators, ${ }^{4}$ micellar reactors ${ }^{5}$ and liquid crystals. ${ }^{6}$ They also have gained increasing interest in the past two decades in the field of glycobiology as tools to probe carbohydrateprotein recognition. Jayaraman and co-workers, reported in 2002 the synthesis and photoisomerisation properties of azobenzene glycoclusters based on lactose and galactose, and they studied their binding behavior towards high-affinity lectin peanut agglutinin (PNA). ${ }^{7}$ Our group reported on the immobilisation of photoresponsive glycoligands of bacterial lectin FimH onto gold surface, as self-assembled monolayers (glyco-SAMs), and also at the surface of human cells. ${ }^{8}$ Hence, 
the orientation of $\alpha$-D-mannoside ligands was reversibly switched by photoirradiation and allowing for the control of specific adhesion of live bacterial cells. ${ }^{8 a-c}$ More recently, we introduced photoresponsive glycoazobenzene macrocycles, which can change their molecular shape and thus their chiroptical or solubility properties. ${ }^{9 a, b}$ In the same direction, J. Xie and colleagues reported azobenzene glycomacrolactones, ${ }^{10}$ and some of which were stereoselectively cyclized by intramolecular glycosylation. ${ }^{11}$

In order to maximise the transmission of geometrical information from the azo hinge to the sugar as well as the chirality transfer from the sugar to the molecular switch, a straightforward connection between both moieties is required. For this purpose, glycosylation of hydroxyazobenzene derivatives is undoubtedly the most obvious reaction over other approaches such as ester or amide bond formation, urea- or thiourea bridging and copper-catalysed azide-alkyne cycloaddition (CUAAC). The direct conjugation of azobenzene to carbohydrate derivatives by glycosylation was previously achieved in our group employing glycosyl acetimidate or thioglycoside donors, ${ }^{8,9 a, 12}$ or under Mitsunobu conditions. ${ }^{9 b, 13}$ In particular, the use of dihydroxyazobenzene (DHAB) as glycosyl acceptor to build azobenzene bis-glycosides resulted in inconsistent results and limited yields. We investigated recently this reaction in detail and concluded that the scope of glycosyl donors and activation methods is very limited..$^{12}$ As well, the use of Mitsunobu reaction for the conjugation of DHAB to the anomeric centre or primary positions of glycosides was highly substrate-dependent and often led to low to moderate yields. ${ }^{13 b}$ In view of the contrasted results obtained with the aforementioned methods, we sought for a reliable conjugation reaction effective on a wide scope of substrates, also including more sophisticated azoswitches such as ortho-substituted azobenzenes ${ }^{14}$ and diazocine, an ortho-ethylene bridged azobenzene. ${ }^{15}$ Such red-shifted azo derivatives are important as they can be addressed solely with visible light and are thereby compatible with biological media. We focused on a strategy based on halogenides (iodide, bromide) of azo derivatives and glycosyl thiols, respectively as electrophilic and nucleophilic partners in a palladium-catalysed Buchwald-Hartwig-Migita cross coupling (Figure 1). ${ }^{16}$ Although azobenzene derivatives bearing sulfide motifs have already been reported, ${ }^{17,18}$ direct thioarylation of azo switches has never been disclosed. Moreover, systematic studies on the influence of sulfur-based substituents on photochromic and thermal relaxation properties still lack, to the best of our knowledge.

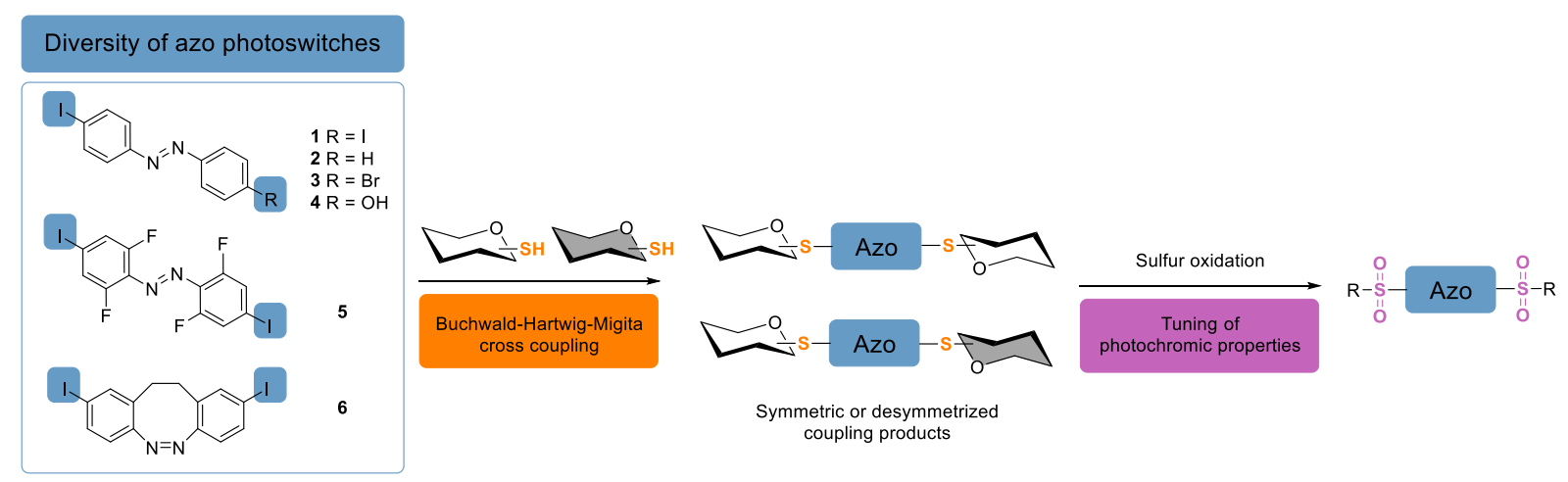

Figure 1: Palladium-catalysed S-arylation of azobenzene derivatives (depicted here with glycosyl thiols) for the preparation of photoswitchable conjugates and subsequent sulfur oxidation enabling photochromic modulation of the glycoconjugates.

The use of isoelectronic sulfur instead of oxygen presents several advantages. First, glycosyl thiols are stable towards mutarotation allowing a complete stereocontrol of the C-1 configuration and thiosaccharides are known to be resistant towards glycosidic bond cleavage mediated by glycosidases. ${ }^{19}$ Second, our synthetic approach allows for straightforward derivatization of orthosubstituted azobenzene or diazocine derivatives, that otherwise require tedious synthetic sequences..$^{14,20,21}$ Third, azobenzene conjugates with a sulfide bridge in para positions may have similar 
photochromic behaviour than para-oxygenated counterparts. Last but not least, the sulfur atom can be oxidised which would modify the electron density in the azobenzene, thus enabling to tune the photochromic properties and the rate of thermal relaxation in the cis state.

We report herein the effective synthesis of various azobenzene conjugate motifs from thioglycosides, as well as other thiol substrates, and diverse azobenzene derivatives, by means of palladium-catalysed thioarylation. In particular, this work includes the preparation of useful red-shifted ABGs and also the streamlined preparation of unsymmetrical conjugates via one-pot procedures. In case of ABGs, subsequent oxidation of the sulfur atom to sulfone or sulfoxide was carried out to investigate the influence of oxidation state on the photochromic and thermal relaxation properties of the switch. Finally, we performed simple spectroscopy experiments to demonstrate the orthogonal photoisomerisation of two distinct derivatives mixed in solution.

\section{Results and discussion}

\section{Bis-functionalisation of $p, p^{\prime}$-diiodoazobenzene derivatives with glycosyl thiols}

Since azobenzene bis-glycosides are key intermediates in the synthesis of photoswitchable glycomacrocycles, we started our investigation by studying the conjugation of $p, p^{\prime}$-diiodoazobenzene derivatives with glycosyl thiols (Scheme 1).

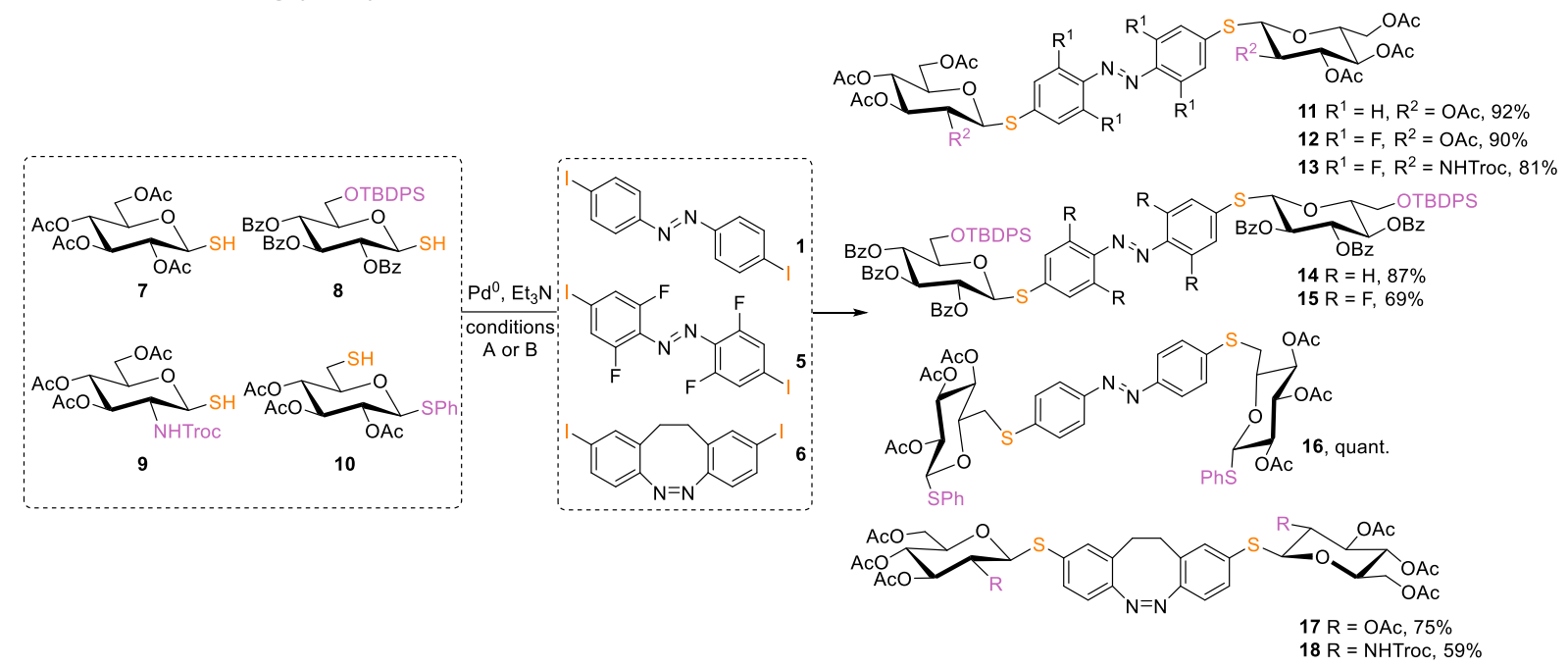

Scheme 1: Preparation of symmetrical azobenzene bis-thioglycosides via thioarylation catalysed by XantphosPdG3 (2mol\%) with excess thiol (2 eq) and $\mathrm{Et}_{3} \mathrm{~N}$ (2 eq) Conditions A: THF, RT; conditions B: $1,4-$ dioxane, $80^{\circ} \mathrm{C}$; compounds 11-16 were prepared according to conditions $A$ whereas compounds $\mathbf{1 7}$ and 18 according to conditions B; TBDPS: tert-butyldiphenylsilyl ; Troc: trichloroethoxycarbonyl.

We chose as electrophiles the azobenzene 1 as a reference, ${ }^{22}$ the tetra-ortho-fluoroazobenzene $\mathbf{5}^{22}$ and diazocine 6. ${ }^{15 \mathrm{c}}$ Tetra-ortho-fluoroazobenzene derivatives present two main features: a red shift of the $n \pi^{*}$ absorption band, thus allowing trans $\rightarrow$ cis switching with visible light irradiation, and a high thermal stability of the metastable cis isomer. ${ }^{14}$ However, to date, only a few functionalized tetraortho-fluoroazobenzenes have been reported, due to multi-step and low yielding synthesis pathway. ${ }^{20}$ As well, the installation of functional hands on diazocine, a promising cyclic azoswitch that exhibits reverse thermodynamic stability and addressability with visible light, is demanding, hence limiting application of this molecular switch. ${ }^{21}$ As nucleophiles we used four differently substituted glucosyl thiols (7-10). We varied the position of the sulfur atom between the anomeric and the primary positions, and also studied the influence of a bulky protecting group. We also used a glucosamine derivative which could pave the way to multifunctional glycoazobenzene macrocycles. In preliminary 
experiments, we compared the two catalytic systems $\mathrm{Pd}(\mathrm{OAc})_{2} /$ Xantphos and the precatalyst Xantphos-PdG3, and observed that the latter was superior, in accordance with previous studies on Sarylation with glycosyl thiols. ${ }^{16}$ We therefore focused on the use of Xantphos-PdG3 for carrying out the present work. Hence, compounds $\mathbf{1 1 - 1 6}$ were obtained at room temperature in 5-20 minutes only using 2 mol\% of catalyst. Both azobenzenes 1 and $\mathbf{5}$ showed excellent conversions and bulky silyl or NTroc protecting groups as in thiols $\mathbf{8}$ and $\mathbf{9}$ did not impair the coupling. Products $\mathbf{1 1 - 1 5}$ were isolated in $69-92 \%$ yield and $\mathbf{1 6}$ that was quantitatively obtained. Conversion of diazocine derivative 6 requested a higher temperature $\left(80^{\circ} \mathrm{C}\right)$ and we thus performed the corresponding reactions in 1,4dioxane to isolate $\mathbf{1 7}$ and $\mathbf{1 8}$ in $\mathbf{7 5 \%}$ and 59\% yield, respectively. Given the excellent results obtained in the preparation of symmetrical motifs, we next intended to focus on the synthesis of unsymmetrical azobenzene conjugates.

\section{Mono-functionalisation of unsymmetrical azobenzenes with different thiols}

In order to assess the scope of the reaction regarding the nature of the thiol substrate, we studied the mono functionalisation of differently substituted $p$-iodoazobenzenes with various thiols (Scheme 2 ). The monosubstituted azobenzene $\mathbf{2}$ was used as a reference compound, and was coupled within minutes to glucosyl thiol 7, affording product $\mathbf{2 3}$ in $90 \%$ yield. Reaction of azobenzene $\mathbf{3}$ with $\mathbf{7}$ gave product $\mathbf{2 4}$ in $87 \%$ yield. We were delighted to observe an excellent chemoselectivity between iodine and bromine, as reported by Messaoudi et al., ${ }^{16 \mathrm{~b}}$ hence allowing for further substitution of the bromide substituent.

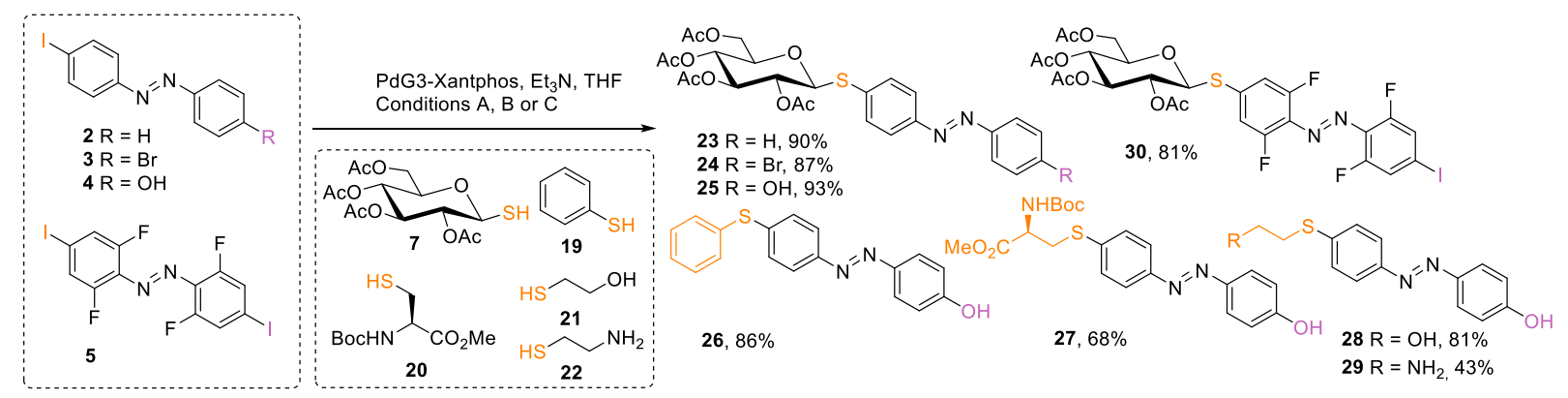

Scheme 2: Mono-functionalisation of differently substituted $p$-iodoazobenzenes with various thiols. Conditions for preparation of compounds 23 - 29: the thiol ( 1 - 1.2 eq) and azobenzene $(1-1.2$ eq) were stirred in THF in presence of PdG3-Xantphos (1 - 5 mol\%) and $\mathrm{Et}_{3} \mathrm{~N}(1-1.4 \mathrm{eq})$ at RT except for 29 which was obtained after heating at $70{ }^{\circ} \mathrm{C}$; conditions for preparation of 30 : excess azobenzene $(2 \mathrm{eq})$ and thiol were stirred at $0{ }^{\circ} \mathrm{C}$ in the presence of 1 mol\% catalyst and $\mathrm{Et}_{3} \mathrm{~N}(1 \mathrm{eq})$.

Azobenzene 4 showed a good tolerability of the reaction towards hydroxyl groups, while providing phenolic conjugates which can be functionalised further by alkylation or glycosylation, for instance. Thus, reaction with $\mathbf{7}$ provided compound $\mathbf{2 5}$ in 93\% yield. Thiophenol 19 smoothly reacted with $\mathbf{4}$ to give product $\mathbf{2 6}$ in $\mathbf{8 6 \%}$ yield. $\mathbf{N}$-Boc-protected cysteine methyl ester $\mathbf{2 0}$ was also readily ligated to $\mathbf{4}$, although longer reaction time was required, affording product $\mathbf{2 7}$ in $68 \%$ yield. Finally, aliphatic thiols bearing competitive hydroxy (21) or amino (22) groups were installed on 4, providing products 28 and 29 in $81 \%$ and $43 \%$ yield, respectively. In case of cysteamine $\mathbf{2 2}$, however, the reaction needed to be performed at $70^{\circ} \mathrm{C}$ and lower yield was obtained due to incomplete conversion. This might be due to the amino group acting as an ancillary ligand somehow impairing the course of the reaction. Importantly, no side-product was observed, thus indicating that $\mathrm{N}$-arylation did not occur. Very interestingly, the lower reaction rate of tetra-ortho-fluoroazobenzene $\mathbf{5}$ allowed us to favor the formation of the mono-coupled product 32 in $81 \%$ yield, by using an excess of azobenzene ( 2 eq) at $0^{\circ} \mathrm{C}$ under dilute conditions, while the bis-conjugated product was isolated in $14 \%$ yield, and $43 \%$ of 
the initial amount of azobenzene was recovered. These conditions would allow the sequential preparation of unsymmetrical azobenzene glycoconjugates starting from $\mathbf{5}$.

Encouraged by these excellent results, we decided to investigate the streamlined preparation of hetero-bis-azobenzene conjugates via sequential and one-pot procedures.

\section{Hetero-bis-functionalisation of azobenzenes with glycosyl thiols}

Starting from the previously synthesised unsymmetrical azobenzene monothioglycosides $\mathbf{2 4}$ and $\mathbf{2 5}$, we prepared the corresponding hetero-bis-glycosides $\mathbf{3 1}$ and $\mathbf{3 2}$ (Scheme 3). Conjugate $\mathbf{3 1}$ was thus obtained in good yield (73\%) by substitution of the para-bromide in $\mathbf{2 4}$, in the presence of XantphosPdG3 at $60{ }^{\circ} \mathrm{C}$. On the other hand, the phenolic glycosyl acceptor 25 was reacted with glycosyl imidate donor 33 (prepared in one step from thiol 7), ${ }^{23}$ using $\mathrm{BF}_{3} . \mathrm{OEt}_{2}$ as glycosylation promoter, to provide compound 32 in $67 \%$ yield.
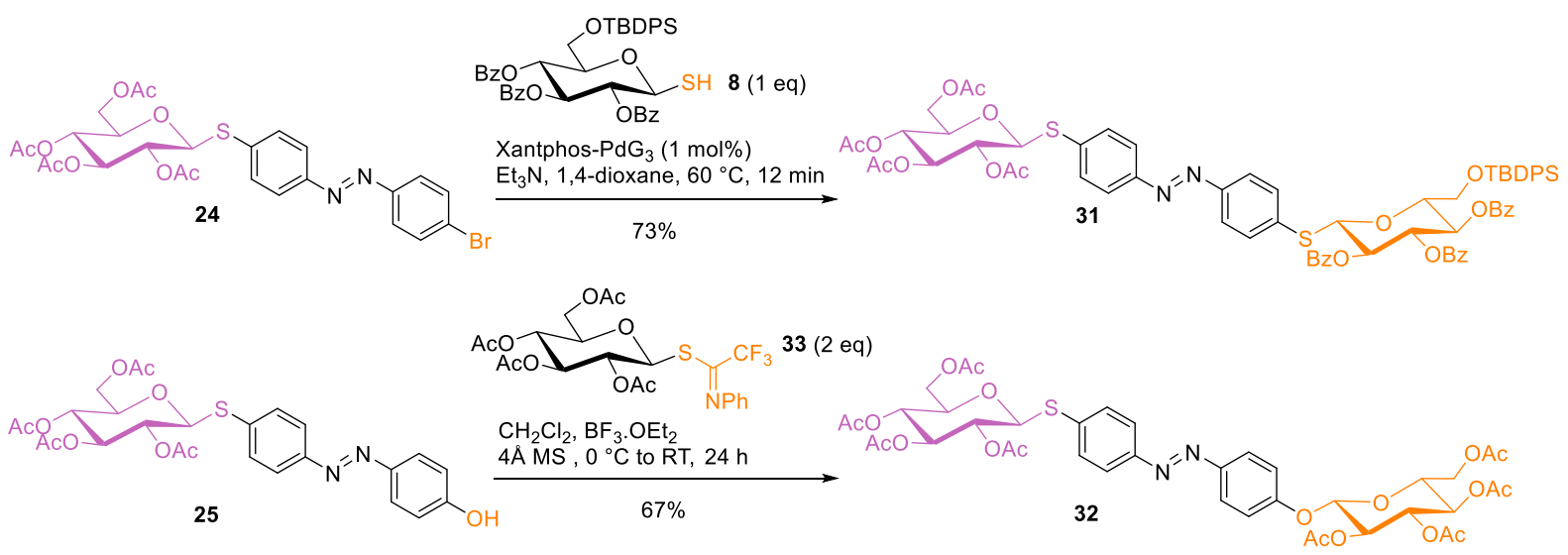

Scheme 3: Preparation of unsymmetrical azobenzene bis-glycoconjugates following two sequential coupling reactions (top), or by sequential coupling and subsequent glycosylation (bottom).

We then turned our attention to one-pot procedures for preparing unsymmetrical azobenzene conjugates (Scheme 4). First of all, we exploited the sharp difference in reactivity between the opposite para-iodo and para'-bromo substituents on $\mathbf{3}$ to perform sequential $S$-arylation reactions in the same vessel. In order to guarantee a proper coupling in the second step, we systematically added the same amount of catalyst as the initial load ( 1 mol\%). Thus 3 was first substituted by thiol $\mathbf{7}$ at room temperature and then by thiol 8 at $60{ }^{\circ} \mathrm{C}$ to provide product 31 in a good yield of $73 \%$. According to a similar procedure, $\mathbf{3}$ was successfully coupled to both $\mathbf{7}$ and the cysteine derivative $\mathbf{2 0}$ to give conjugate 34 in $70 \%$ yield. Next, we took advantage of the lower reactivity of $\mathbf{5}$ to carry out a one-pot synthesis in which stoichiometric amounts of $\mathbf{2}$ and thiol $\mathbf{7}$ were reacted at $-10^{\circ} \mathrm{C}$, followed by addition of excess thiol $\mathbf{8}$ to afford $\mathbf{3 5}$ in a fair yield of $51 \%$. Finally, we prepared the conjugate $\mathbf{3 6}$, bearing a thioacetateterminated linker, suitable for the fabrication of glyco-SAMs on gold surface. Azobenzene $\mathbf{4}$ was first coupled with thiol $\mathbf{7}$ at room temperature then, alkylation of the phenol with tosylated spacer $\mathbf{3 7}$ in DMF at $80^{\circ} \mathrm{C}$ yielded $57 \%$ of the desired product 36 .

With a collection of azobenzene $S$-glycosides in hand, we focused our next efforts on the preparation of sulfoxide and sulfone derivatives through oxidation of the sulfur atom(s).

\section{Oxidations at sulfur atom}

We used meta-chloroperbenzeoic acid ( $m C P B A$ ) to oxidise sulfur bridges into sulfoxides or sulfones on a selection of previously synthesised azobenzene glycoconjugates (Scheme 5). In fact, electron withdrawing substituents in para positions of azobenzenes influence both $\pi \rightarrow \pi^{*}$ and $n \rightarrow \pi^{*}$ transitions, ${ }^{14 d, 18 d, 24}$ as well as relaxation kinetics of the cis isomer..$^{25}$ We therefore expected an 
hypsochromic shift of the $\pi \rightarrow \pi^{*}$ band, compared to the parent sulfide derivative, and also an increased separation of the $n \rightarrow \pi^{*}$ bands of each isomer, as reported in case of ortho-substituted azobenzenes. ${ }^{14}$ Oxidation of monosubstituted compound $\mathbf{2 3}$ with a slight excess of $m C P B A$ in dichloromethane afforded a mixture of sulfoxide 38 a and sulfone $\mathbf{3 8 b}$ which were isolated in $43 \%$ and $53 \%$ yield, respectively. Mixed conjugates having a sulfone and a sulfide at each end were prepared following a two-step sequence. Hence, oxidation of compound $\mathbf{2 4}$ was achieved, affording sulfone $\mathbf{3 9}$ in $\mathbf{8 7 \%}$ yield, and subsequent coupling with thiol $\mathbf{7}$ afforded compound $\mathbf{4 0}$ in $\mathbf{4 6 \%}$ yield. In the same manner, sulfone $\mathbf{4 1}$ was obtained in $68 \%$ yield from 30, and next reacted with thiol $\mathbf{7}$ to provide $\mathbf{4 2}$ in $59 \%$ yield. Finally, the complete oxidation of molecules $\mathbf{1 1}$ and $\mathbf{1 3}$ afforded disulfone derivatives $\mathbf{4 3}$ and $\mathbf{4 4}$ in $\mathbf{7 9 \%}$ and $78 \%$ yield, respectively.

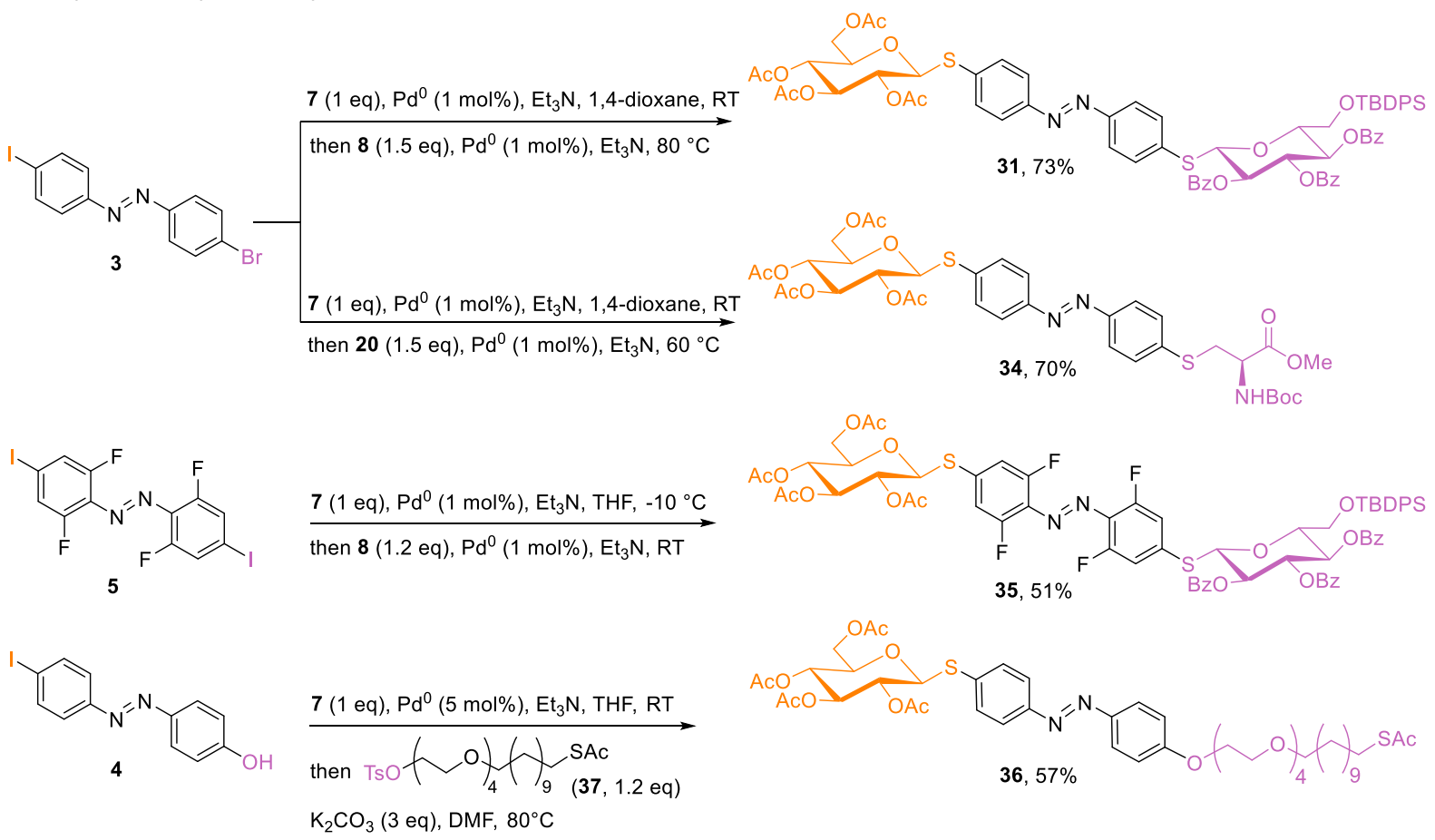

Scheme 4: Preparation of unsymmetrical azobenzene hetero-bis-conjugates in a one-pot fashion. $\mathrm{Pd}^{0}$ : XantphosPdG3.
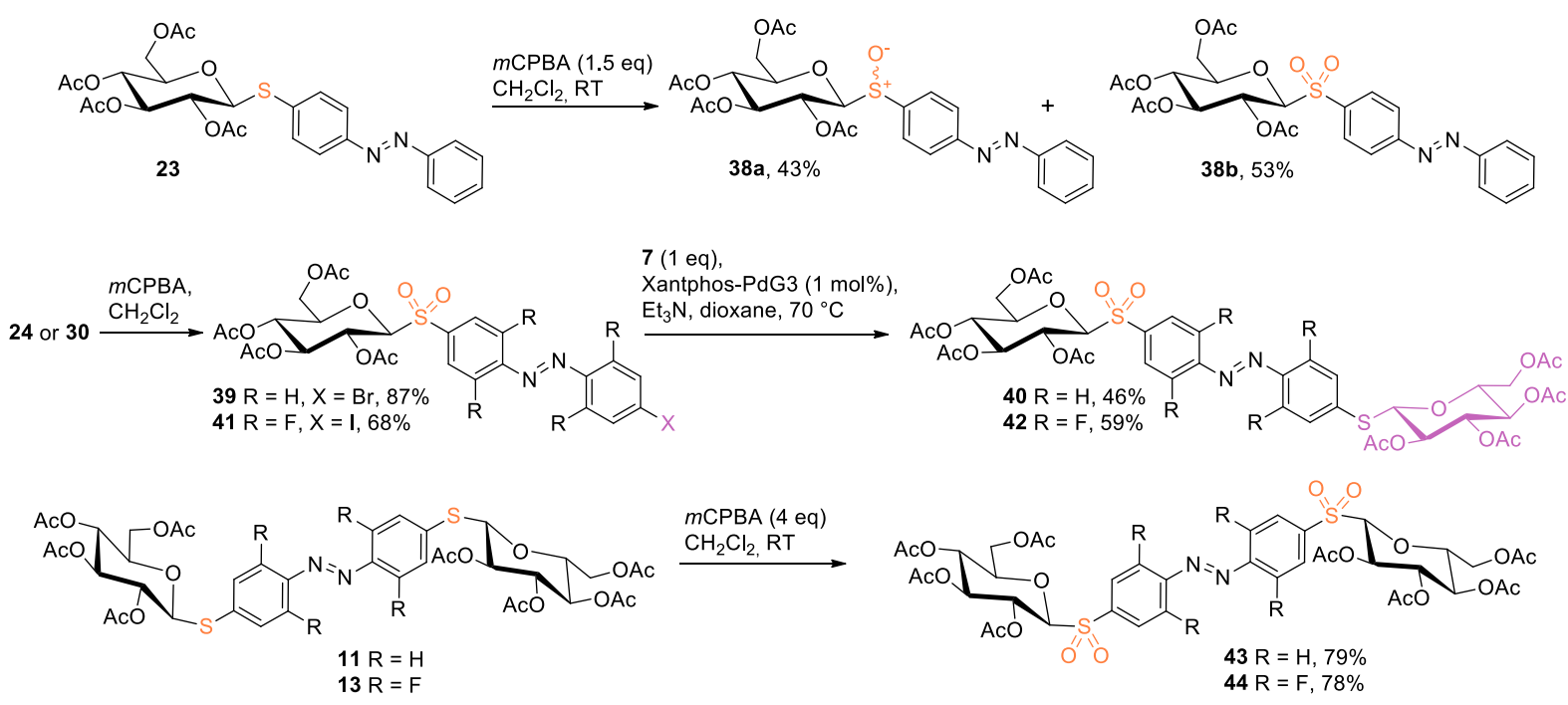

Scheme 5: $m$ CPBA-mediated oxidations at sulfur centre to afford sulfoxide (38a) or sulfone $(38 b, 43,44)$ derivatives. Mixed products bearing both sulfide and sulfone functions $(\mathbf{4 0}, \mathbf{4 2})$ were synthesized in a sequential fashion. 


\section{Photochromic properties of the synthesised azobenzene glycoconjugates}

Among the synthesised glycoconjugates, we selected 9 azobenzene glycoconjugates (Table 1 ) in order to investigate the photochromic properties and the relaxation of the metastable isomers. The set was defined in such a way that we could evaluate the influence of substitution patterns in para and ortho positions of the azobenzene core as well as the impact of the sulfur oxidation state. The main spectroscopic data are listed in Table 1 and Figure 2 shows absorption spectra of the assessed molecules in DMSO.

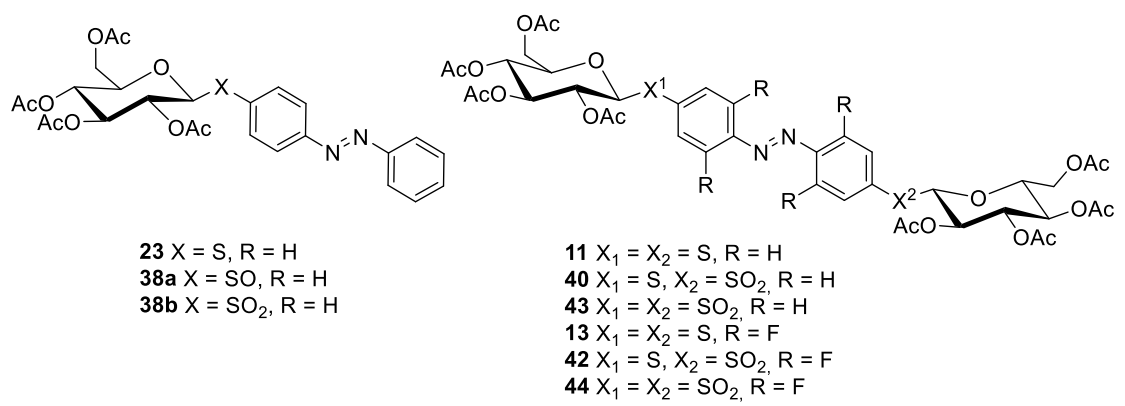

Table 1. Spectroscopic data of selected azobenzene thioglycosides: maxima $\left(\lambda_{\max }\right)$ of $\pi \rightarrow \pi^{*}$ and $n \rightarrow \pi^{*}$ transitions of trans and cis isomers, ${ }^{[a]}$ with the corresponding separation of the $n \rightarrow \pi^{*}$ bands $\left(\Delta \lambda_{n \rightarrow \pi^{*}}\right)$ in each isomeric state, composition of the PSS mixtures at different wavelengths of irradiation ${ }^{[b]}$ and half-lives of thermal relaxation. ${ }^{[c]}$

\begin{tabular}{|c|c|c|c|c|c|c|c|c|c|c|}
\hline Entry & Compound & $\begin{array}{c}\lambda_{\max } \\
\pi \rightarrow \pi^{*} \\
\text { trans } \\
{[\mathrm{nm}]}\end{array}$ & $\begin{array}{c}\lambda_{\max } \\
n \rightarrow \pi^{*} \\
\text { trans } \\
{[\mathrm{nm}]}\end{array}$ & $\begin{array}{c}\lambda_{\max } \\
n \rightarrow \pi^{*} \\
\text { cis } \\
{[\mathrm{nm}]}\end{array}$ & $\begin{array}{c}\Delta \lambda_{n \rightarrow \pi^{*}} \\
{[\mathrm{~nm}]}\end{array}$ & $\begin{array}{c}\text { PSS } \\
365^{[b]}\end{array}$ & $\begin{array}{c}\text { PSS } \\
435^{[b]}\end{array}$ & $\begin{array}{c}\text { PSS } \\
520^{[b]}\end{array}$ & $\begin{array}{c}\text { PSS } \\
590^{[d]}\end{array}$ & $\mathbf{t}_{1 / 2}$ cis \\
\hline 1 & 23 & 351 & 441 & 439 & 2 & $5 / 95$ & $78 / 22$ & - & - & $10 \mathrm{~h}$ \\
\hline 2 & $38 a$ & 334 & 451 & 434 & 17 & $10 / 90$ & $84 / 16$ & - & - & $20 \mathrm{~h}$ \\
\hline 3 & $38 b$ & 327 & 453 & 432 & 21 & $32 / 68$ & $87 / 13$ & - & - & $2.1 \mathrm{~h}$ \\
\hline 4 & 11 & 374 & ND & 445 & - & $14 / 86$ & $74 / 26$ & $82 / 18$ & $95 / 5^{d}$ & $3.0 \mathrm{~h}$ \\
\hline 5 & 40 & 366 & ND & ND & - & $31 / 69$ & $86 / 14$ & $80 / 20$ & $86 / 14^{d}$ & $1.4 \mathrm{~h}$ \\
\hline 6 & 43 & 317 & 464 & ND & - & - & - & - & - & - \\
\hline 7 & 13 & 369 & 460 & 433 & 27 & $20 / 80$ & $80 / 20$ & $30 / 70$ & $16 / 84^{d}$ & $96 \mathrm{~h}$ \\
\hline 8 & 42 & 358 & 464 & 429 & 35 & $32 / 68$ & $86 / 14$ & $32 / 68$ & $8 / 92^{d}$ & $177 \mathrm{~h}$ \\
\hline 9 & $44^{[\mathrm{e}]}$ & 310 & 472 & 423 & 49 & $97 / 3$ & $91 / 9$ & $28 / 72$ & $9 / 91^{d}$ & $31 \mathrm{~min}$ \\
\hline
\end{tabular}

[a] UV-Vis spectra were recorded in DMSO $(50 \mu \mathrm{M})$ at $25^{\circ} \mathrm{C}$; ND: not determined because of the overlap between the $\pi \rightarrow \pi^{*}$ and $n \rightarrow \pi^{*}$ transition bands; [b] composition at the PSS were measured by NMR in $d_{6}$-DMSO ( $1 \mathrm{mM}$ ), after $2 \mathrm{~min}$ irradiation at the appropriate wavelength; mixtures at the PSS equilibrium are given as: $\%$ (trans)/\%(cis); [c] the half-lives of cis isomers were measured by UV-Vis spectroscopy in DMSO (50 $\mu \mathrm{M})$ at 40 ${ }^{\circ} \mathrm{C}$; [d] after $2 \mathrm{~min}$ irradiation with UV $(365 \mathrm{~nm})$ or green $(520 \mathrm{~nm})$ light, the sample was irradiated for 5-20 min with orange $\left(590 \mathrm{~nm}\right.$ ) light; [e] PSS were measured by NMR in $d_{3}-\mathrm{MeCN}(1 \mathrm{mM})$ after 2 min irradiation at the appropriate wavelength.

The first subset of molecules, that comprises the monosubstituted derivatives $\mathbf{2 3}$, 38a and $\mathbf{3 8} \mathbf{b}$, provided a good basis to evaluate the influence of sulfur oxidation on the photochromic behaviour. In line with a previously reported $O$-glycoside counterpart, ${ }^{26} 23$ in the ground state shows two absorption maxima at $351 \mathrm{~nm}$ and $441 \mathrm{~nm}$, respectively corresponding to $\pi \rightarrow \pi^{*}$ and $n \rightarrow \pi^{*}$ transitions (Table 1entry 1, Fig. 1a). Increasing the oxidation state of the sulfur atom results in a hypsochromic shift of the $\pi \rightarrow \pi^{*}$ band in case of trans isomers, the effect being stronger for the sulfone derivative (Table 1, entry 2 and 3, Fig. 1a). Photostationary states (PSS) containing high amounts of cis isomer are reached upon irradiation with UV light (365 nm), for $\mathbf{2 3}$ and 38a. In contrast, $68 \%$ of cis-38b is obtained after photoisomerization at the same wavelength, indicating a red shift of the cis $\pi \rightarrow \pi^{*}$ band and thus a larger overlap of the respective $\pi \rightarrow \pi^{*}$ bands of the trans and cis isomers. In addition, sulfur oxidation leads to a bathochromic shift of the $n \rightarrow \pi^{*}$ transition in the trans isomer whereas it gets slightly shifted 
to lower wavelengths in the cis form (Fig. 1d). Consequently, compared to the sulfide 23, the $n \rightarrow \pi^{*}$ band separation is greater in the oxidated counterparts although not sufficient to achieve trans $\rightarrow$ cis isomerisation with visible light. Increase of sulfur oxidation state also strongly impacts the thermal relaxation rate of the cis isomer: half-life drops by a factor 5 from the sulfide $\mathbf{2 3}(10 \mathrm{~h})$ to the sulfone 38b (2.1 h) while it is $20 \mathrm{~h}$ in case of the sulfoxide 38a (Table 1, entry 1-3).

Absorption spectra of $p, p^{\prime}$-difunctionalized compounds $\mathbf{1 1}, \mathbf{4 0}, \mathbf{4 3}$ and $\mathbf{1 3}, \mathbf{4 2 ,} \mathbf{4 4}$ show a similar trend as for monosubstituted derivatives. Whereas trans isomers of homo bis-thioglucosides $\mathbf{1 1}$ and $\mathbf{1 3}$ exhibit absorption maxima around $370 \mathrm{~nm}$ for the $\pi \rightarrow \pi^{*}$ band (Table 1, entry 7 and 10, Fig. 1b and 1c), the corresponding $\lambda_{\max }$ decreases moderately for mixed sulfide/sulfone conjugates $\mathbf{4 2}$ and $\mathbf{4 4}$ (Table 1, entry 9 and 12). Oxidation of both sulfide bridges to sulfones (compounds $\mathbf{4 3}$ and $\mathbf{4 4}$ ) causes a dramatic hypsochromic shift (over $50 \mathrm{~nm}$ compared to $\mathbf{1 1}$ and 13, respectively) of the same electronic transition (Table 1, entry 9 and 12). Variation of the $n \rightarrow \pi^{*}$ band cannot be properly observed with molecules 11 and $\mathbf{4 0}$ due to a strong overlap with the $\pi \rightarrow \pi^{*}$ transition (Fig. 1e). However, examination of the orthofluoroazobenzenes 13, 42, 44 (Fig. 1f) provides information that comply with what observed for the monosubstituted conjugates: upon sulfur oxidation, the $n \rightarrow \pi^{*}$ band in trans isomers undergoes a red shift while it is blue-shifted in cis isomers. As a consequence, the gap between the two bands, already stemming from the ortho-fluoro groups, gets increased up to $49 \mathrm{~nm}$ after sulfur oxidation. This result is not surprising as it was reported that electron withdrawing groups in para to the azo bond improve the separation of $n \rightarrow \pi^{*}$ bands. ${ }^{15 a, b}$

Irradiation of 11, 40, 13 and $\mathbf{4 2}$ with light of $365 \mathrm{~nm}$ affords cis-rich mixtures in the PSS. As observed before, the amount of cis isomer decreases in case of $\mathbf{4 0}$ and $\mathbf{4 2}$ probably due to a red shift of the $\pi \rightarrow \pi^{*}$ in the cis form. When the same molecules are exposed to blue light $(435 \mathrm{~nm})$, the trans isomer dominates in the PSS mixture (74-86\%). Addressing 13 and 42 with green light $(520 \mathrm{~nm})$ respectively afforded $70 \%$ and $68 \%$ of cis isomer in the equilibrium mixture whereas $\mathbf{1 1}$ and $\mathbf{4 0}$ were mostly converted into trans at the same wavelength. Due to the strong blue shift of the $\pi \rightarrow \pi^{*}$ band, it is not possible to isomerise disulfone 43 using UV ( $365 \mathrm{~nm}$ ) nor visible light and we observed a scarce change in the absorption spectrum after minutes of exposure to UV-B $(280-315 \mathrm{~nm})$. Nevertheless, the orthofluoro counterpart $\mathbf{4 4}$ presents interesting photochromic features. In fact, effective conversion to the cis isomer ( $72 \%$ in the PSS) is achieved by illumination with green light (Table 1, entry 12 ) and $97 \%$ and 91\% trans-44 are obtained, respectively upon UV- and blue light irradiation.

We also looked at the possibility to drive isomerization with orange light $(590 \mathrm{~nm})$, which is beneficial for using azobenzene conjugates in biological studies. Although the molar extinction in the near red range may be quite weak, such effective photoisomerization performed by addressing the tail of the $n \rightarrow \pi^{*}$ band has been already reported in case of ortho-substituted azobenzenes. ${ }^{18 d, 27,28}$ To our delight, trans $\rightarrow$ cis switching is effective upon irradiation of compounds 13,42 and 44 at $590 \mathrm{~nm}$. In addition, the amount of cis isomer obtained in the PSS at $590 \mathrm{~nm}$ is larger (84 - 92\%) than after photoisomerization with light of lower wavelengths (see Table 1). We observed that the time needed to reach the PSS at $590 \mathrm{~nm}$ is dependent on the composition of the starting trans/cis mixture: the higher the amount of trans, the longer the irradiation time (see ESI). Exposure to orange light was also carried out with compounds $\mathbf{1 1}$ and $\mathbf{4 0}$, and in both cases, the extent of trans isomer is significantly increased after irradiation times in the same range than those needed for the fluorinated derivatives. Regarding the thermal return of metastable cis isomers at $40{ }^{\circ} \mathrm{C}$, cis-40 relaxes nearly two-fold faster than cis-11, with respective half-lives of $3 \mathrm{~h}$ and $1.4 \mathrm{~h}$. As expected, cis-13 is thermally stable $\left(\mathrm{t}_{1 / 2}=96\right.$ h) whereas the cis-disulfone $\mathbf{4 4}$ relaxes over 180 -fold quicker ( $t_{1 / 2}=31 \mathrm{~min}$ ). Surprisingly, the half-life of the hetero conjugate cis-42 was found to be almost twice as much as the one of cis-13, with a value of $177 \mathrm{~h}$.

Finally, diazocine bis-glycoconjugate $\mathbf{1 7}$ was evaluated also in DMSO (see ESI for details). Irradiation of $\mathbf{1 7}$ at $\mathbf{4 0 0} \mathrm{nm}$, yielded $71 \%$ of trans isomer in the PSS while cis-17 was quantitatively recovered upon exposure to green or orange light. Moreover, a half-life of $22 \mathrm{~min}\left(40^{\circ} \mathrm{C}\right)$ was measured for trans-17. 
Interestingly, the trans $\rightarrow$ cis isomerisation process can also be achieved quantitatively with red (630, $660 \mathrm{~nm}$ ) and even far-red light $(690 \mathrm{~nm})$, although longer irradiation time was required in the last case. To the best of our knowledge, only red-shifted diazocines, containing an hetero atom in the bridge, showed similar features. ${ }^{15 b}$ Hence, our thioarylation approach allows for streamlined preparation of functional and red light-switchable diazocines in only three steps.
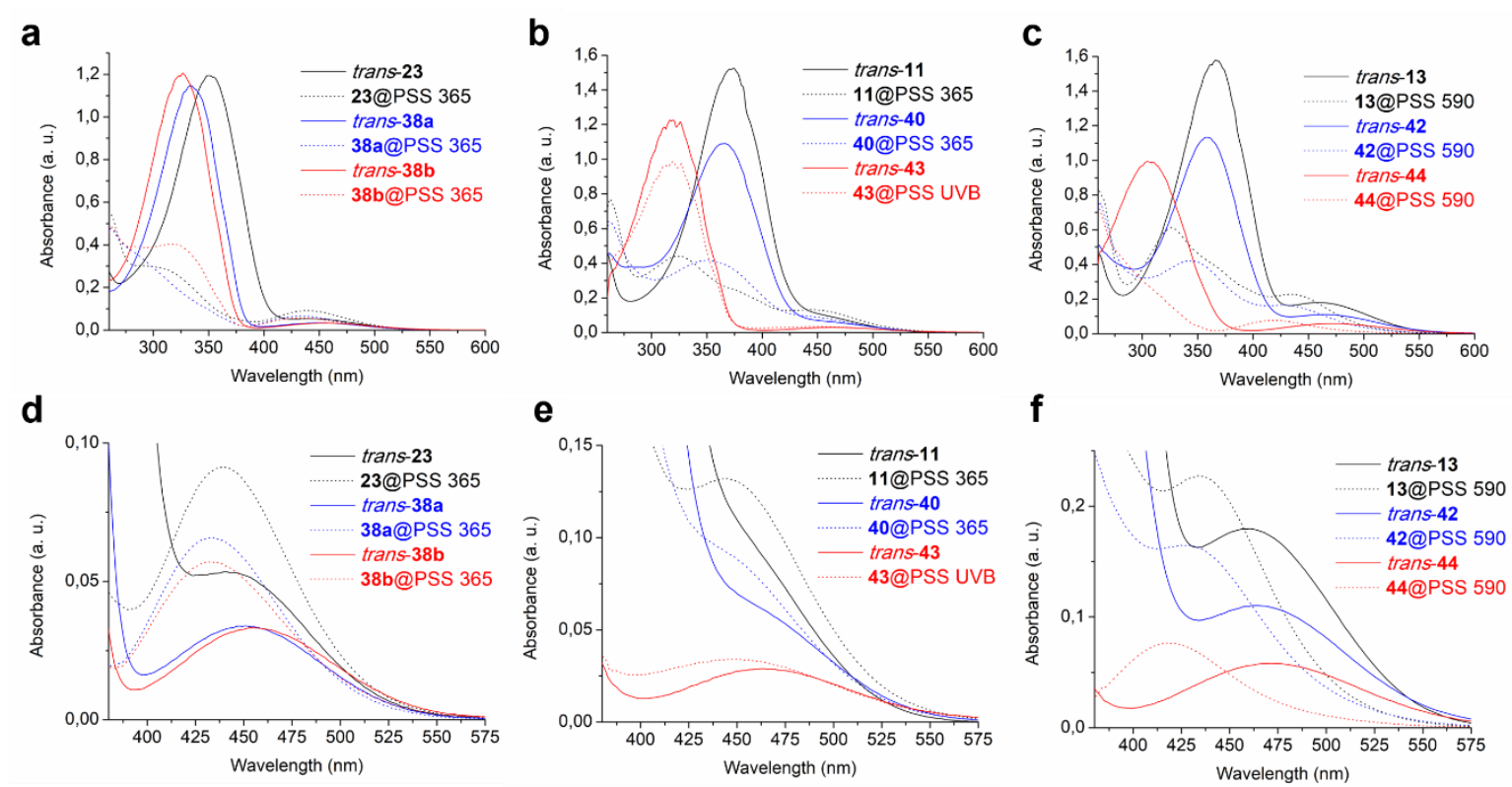

Figure 2. Overlays of absorption spectra and corresponding expansions in the area of the $n \rightarrow \pi^{*}$ transitions for compounds 23, 38a, and $\mathbf{3 8 b}(\mathrm{a}, \mathrm{d}), \mathbf{1 1}, \mathbf{4 0}$, and $\mathbf{4 3}$ (b, e), 13, 42, and $\mathbf{4 4}$ (c, f); solid lines: trans isomers, dotted lines: cis-rich photostationary states after irradiation at an appropriate wavelength; spectra were recorded in DMSO $(50 \mu \mathrm{M})$ at $25^{\circ} \mathrm{C}$.

\section{Orthogonal photoswitching in mixtures}

Based on the photoisomerization results reported here above, we anticipated the possibility to achieve orthogonal switching by irradiating mixtures of two distinct conjugates in solution. Such a feature is of great interest for controlling two distinct parameters in complex light-responsive molecular systems. ${ }^{27,29}$ In fact, it may be difficult to orthogonally achieve each of the four possible isomers only using light triggers, ${ }^{30}$ and systems solely fueled by light require fine-tuning of irradiation conditions. ${ }^{27,29}$ Hence, we selected two pairs of relevant molecules that may be addressed at least with light of three different wavelengths. We investigated by NMR spectroscopy equimolar mixtures of compounds $\mathbf{1 1}$ and 44, on the one hand, and of $\mathbf{1 7}$ and 44, on the other hand. Hence it was expected to switch between trans/trans, cis/trans and trans/cis mixtures, in case of the pair 11/44. In an alternative fashion, the pair 17/44 would allow the selection among cis/trans, cis/cis and trans/trans states. By operating with light in the near UV - green range, it was possible to reach PSS mixtures with over $70 \%$ of the major isomer for each of the two compounds present in solution, thus achieving the purpose described here above (see Table 2). The trans/cis ratios observed at each PSS in case of the mixtures were found to be very similar to those measured with the single molecules. This indicates that, at the concentration chosen for these experiments, the photoswitchable conjugates may not disturb photochromic properties of each other. 


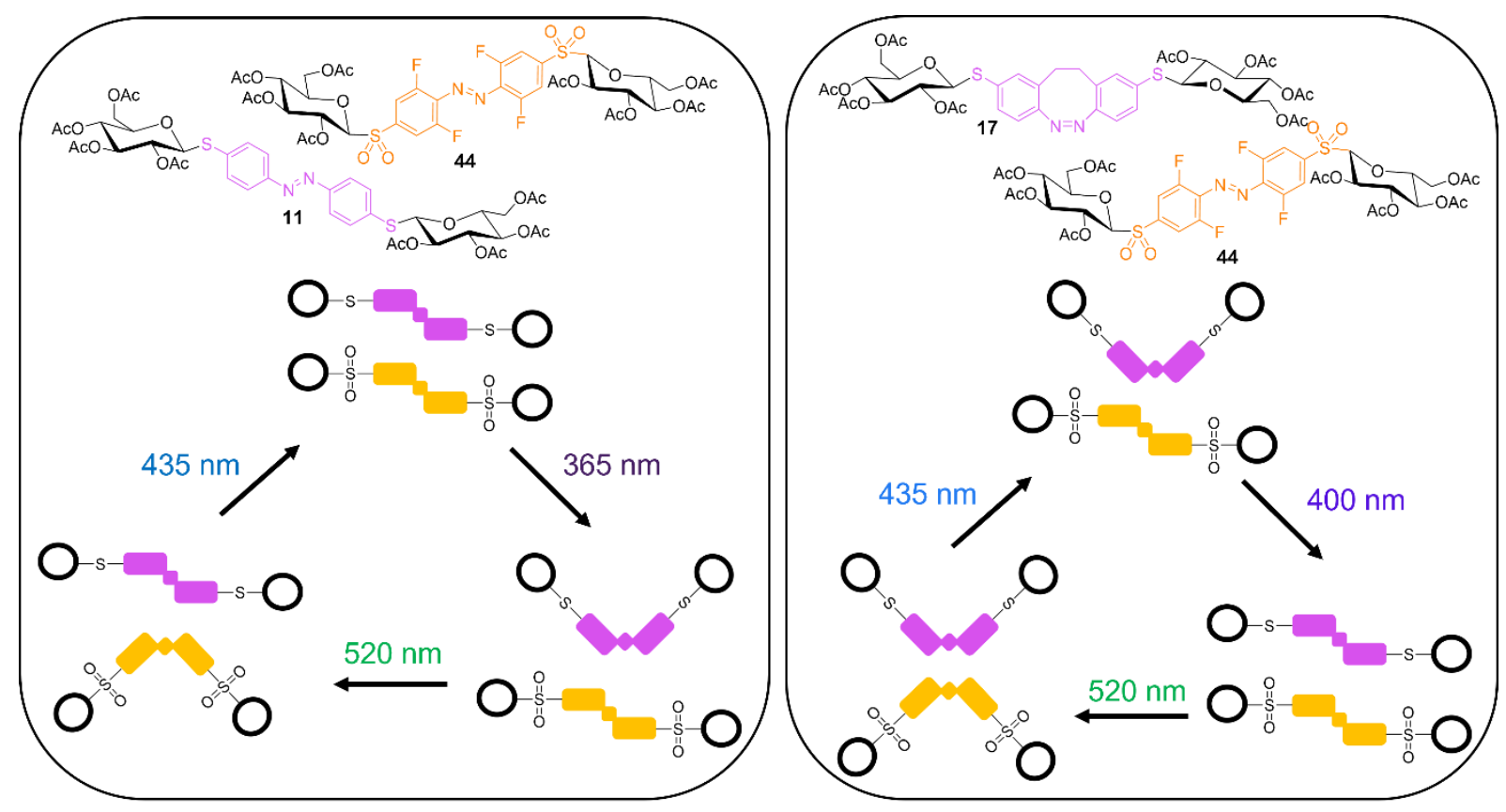

Figure 3. Orthogonal photoisomerization of azobenzene or diazocine glycoconjugates in solution mixtures with light of three distinct wavelengths. Two pairs of conjugates were investigated: 11/44 and 17/44.

Table 2. Isomer ratios measured by ${ }^{1} \mathrm{H}$ NMR spectroscopy for equimolar mixtures $11 / 44$ and $17 / 44$, in $\mathrm{d}_{3}-\mathrm{MeCN}$ $(1 \mathrm{mM}, 300 \mathrm{~K})$, after irradiation with light of $365 \mathrm{~nm}, 400 \mathrm{~nm}, 435 \mathrm{~nm}$ or $520 \mathrm{~nm}$.

\begin{tabular}{|c|c|c|c|c|}
\hline & $365 \mathrm{~nm}$ & $400 \mathrm{~nm}$ & $435 \mathrm{~nm}$ & $520 \mathrm{~nm}$ \\
\hline $\mathbf{1 1}$ trans:cis & $17: 83$ & - & $78: 22$ & $78: 22$ \\
\hline $\mathbf{4 4}$ trans:cis & $97: 3$ & - & $90: 10$ & $27: 73$ \\
\hline $\mathbf{1 7}$ trans:cis & - & $72: 28$ & $29: 71$ & $>1: 99$ \\
\hline $\mathbf{4 4}$ trans:cis & - & $97: 3$ & $91: 9$ & $27: 73$ \\
\hline
\end{tabular}

\section{Conclusion}

In conclusion, we disclosed a simple, yet effective, direct functionalisation of azobenzenes derivatives, based on a palladium-catalysed thioarylation reaction. We demonstrated the utility of this method through the synthesis of a wide scope of conjugates, especially challenging disymmetrized compounds via straightforward one-pot procedures. Although we mostly focused on the use of glycosyl thiols, we showed that the coupling is compatible with other kind of thiols and also tolerates other unprotected functional groups. Importantly, we could easily functionalise valuable red-shifted photoswitches such as ortho-fluorinated azobenzene and diazocine, without the need of tedious reaction sequences. In addition to our synthesis work, we investigated the photochromic and thermal relaxation properties of mono- and disubstituted ABGs and the influence of sulfur oxidation on these features. All in all, the presence of sulfur-based groups (sulfide, sulfoxide and sulfone) in para positions imparts favourable photoisomerization behaviour with efficient switching in both cis/trans directions upon exposure to light of appropriate wavelength. Especially, the ortho-fluorinated conjugates can be effectively addressed solely with visible light, including trans $\rightarrow$ cis isomerization by irradiation with light of 590 $\mathrm{nm}$. In addition, increasing sulfur oxidation state allows for tuning absorption maxima as well as thermal relaxation rate of the metastable cis isomer. Finally, we demonstrated the selective photoisomerization in solutions containing mixtures of two distinct ABGs.

With this efficient functionalisation method in hand, our next efforts will be focused on the preparation of photoresponsive carbohydrate ligands for lectin recognition and also on the synthesis of 
azobenzene glycomacrocycles. The modularity of our approach will allow the elaboration of complex molecular systems, comprising several distinct photoswitches, in which more than one parameter can be controlled with light. We believe that this work provides new useful tools for the design of photoswitchable molecules used in chemical, material and biological sciences.

\section{References}

1. (a) A. A. Beharry, G. A. Woolley, Chem. Soc. Rev. 2011, 40, 4422-4437; (b) W. Szymanski, J. M. Beierle, H. A. V. Kistemaker, W. A. Velema, B. L. Feringa, Chem. Rev. 2013, 113, 6114-6178.

2. (a) H. M. D. Bandara, S. C. Burdette, Chem. Soc. Rev. 2012, 41, 1809-1825; (b) E. Merino, Chem. Soc. Rev. 2011, 40, 3835-3853; (c) D. Bléger, S. Hecht, Angew. Chem. Int. Ed. 2015, 54, 1133811349; (d) M. Dong, A. Babalhavaeji, S. Samanta, A. A. Beharry, G. A. Woolley, Acc. Chem. Res. 2015, 48, 2662-2670.

3. Y. Hu, R. F. Tabor, B. L. Wilkinson, Org. Biomol. Chem. 2015, 13, 2216-2225.

4. M. J. Clemente, R. M. Tejedor, P. Romero, J. Fittreman, L. Oriol, RSC Adv. 2012, 2, 1141911431.

5. N. Drillaud, E. Banaszak-Léonard, I. Pezron and C. Len, J. Org. Chem., 2012, 77, 9553-9561.

6. N. Laurent, D. Lafont, F. Dumoulin, P. Boullager, G. Mackenzie, P. H. J. Kouwer, J. W. Gooodby, J. Am. Chem. Soc. 2003, 125, 15499-15506.

7. O. Srinivas, N. Mitra, A. Surolia, N. Jayaraman, J. Am. Chem. Soc. 2002, 124, 2124-2125.

8. (a) T. Weber, V. Chandrasekaran, I. Stamer, M. B. Thygesen, A. Terfort, T. K. Lindhorst, Angew. Chem. Int. Ed. 2014, 53, 14583-14586; (b) L. Möckl, A. Müller, C. Bräuchle, T. K. Lindhorst Chem. Commun. 2016, 52, 1254-1257; (c) G. Despras, L. Möckl, A. Heitmann, I. Stamer, C. Bräuchle, T. K. Lindhorst, ChemBioChem 2019, 20, 2373-2382; (d) E. Fast, A. Schlimm, I. Lautenschläger, K. U. Clausen, T. Strunskus, C. Spormann, T. K. Lindhorst, F. Tuczek, Chem. Eur. J. 2020, 26, 485-501.

9. (a) G. Despras, J. Hain, S. O. Jaeschke, Chem. Eur. J. 2017, 23, 10838-10847; (b) J. Hain, G. Despras, Chem. Commun. 2018, 54, 8563-8566.

10. (a) C. Lin, S. Maisonneuve, R. Métivier, J. Xie, Chem. Eur. J. 2017, 23, 14996-15001 ; (b) C. Lin, S. Maisonneuve, C. Theulier, J. Xie, Eur. J. Org. Chem., 2019, 1770-1777.

11. C. Lin, J. Jiao, S. Maisonneuve, J. Mallétroit, J. Xie, Chem. Commun. 2020, 56, 3261-3264.

12. J. Berry, G. Despras, T. K. Lindhorst, RSC Adv. 2020, 10, 17432-17437.

13. (a) J. Hain, V. Chandrasekaran, T. K. Lindhorst, Isr. J. Chem. 2015, 55, 383-386; (b) J. Hain, P. Rollin, W. Klaffke, T. K. Lindhorst, Beilstein J. Org. Chem. 2018, 14, 1619-1636.

14. (a) A. A. Beharry, O. Sadovski, G. A. Wolley, J. Am. Chem. Soc. 2011, 133, 19684-19687; (b) Bléger, J. Schwarz, A. M. Brouwer, S. Hecht, J. Am. Chem. Soc. 2012, 134, 20597-20600; (c) S. Samanta, A. A. Beharry, O. Sadovski, T. McCormick, A. Babalhavaeji, V. Tropepe, G. A. Wolley, J. Am. Chem. Soc. 2013, 133, 49, 19684-19687; (d) C. Knie, M. Utecht, F. Zhao, H. Kulla, S. Kovalenko, A. M. Brouwer, P. Saalfrank, S. Hecht, D. Bléger, Chem. Eur. J. 2014, 20, 1649216501.

15. (a) R. Siewertsen, H. Neumann, B. Buchheimstehn, R. Herges, C. Näther, F. Renth, F. Temps, J. Am. Chem. Soc. 2009, 131, 43, 15594-15595; (b) M. Hammerich, C. Schütt, C. Stähler, P. Lentes, F. Röhricht, R. Höppner, R. Herges, J. Am. Chem. Soc. 2016, 138, 13111-13114; (c) M. S. Maier, K. Hüll, M. Reynders, B. S. Matsuura, P. Leippe, T. Ko, L. Schäffer, D. Trauner, J. Am. Chem. Soc. 2019, 141, 17295-17304.

16. (a) E. Brachet, J. D. Brion, M. Alami, S. Messaoudi, Adv. Synth. Catal. 2013, 355, 2627-2636; (b) A. Bruneau, M. Roche, A. Hamze, J. D. Brion, M. Alami, S. Messaoudi, Chem. Eur. J. 2015, 21, 8375-8379. 
17. (a) R. K. Burkhard, R. D. Bauer, D. H. Klaassen, Biochemistry 1962, 1, 819-827; (b) D. Escudero, S. Trupp, B. Bussemer, G. J. Mohr, L. González, J. Chem. Theory Comput. 2011, 7, 1062-1072; (c) R. Wang, Z. Cheng, X. Deng, W. Zhao, Q. Li, Z. Li, J. Mater. Chem. C 2020, 8, 6380-6387.

18. (a) T. Chen, K. Igarashi, N. Nakagawa, K. Yamane, T. Fujii, H. Asanuma, M. Yamashita, J. Photochem. Photobiol. A 2011, 223, 119-123; (b) H. Nishioka, X. Liang, T. Kato, H. Asanuma, Angew. Chem. Int. Ed. 2012, 51, 1165-1168; (c) S. Samanta, T. McCormick, S. K. Schmidt, D. S. Seferos, G. A. Woolley, Chem. Commun. 2013, 49, 10314-10316; (d) L. N. Lameijer, S. Budzak, N. A. Simeth, M. J. Hansen, B. L. Feringa, D. Jacquemin, W. Szymanski, Angew. Chem. Int. Ed. 2020, 59, 21663-21670.

19. H. Driguez, ChemBioChem, 2001, 2, 311-318.

20. (a) A. Rullo, A. Reiner, A. Reiter, D. Trauner, E. Y. Isacoff, G. A. Woolley, Chem. Commun. 2014, 50, 14613-14615; (b) S. lamsaard, E. Anger, S. J. Aßhoff, A. Depauw, S. P. Fletcher, N. Katsonis, Angew. Chem. Int. Ed. 2016, 55, 9908-9912; (c) P. Pfaff, K. T. G. Samarasinghe, C. M. Crews, E. M. Carreira, ACS Cent. Sci. 2019, 5, 1682-1690.

21. (a) S. Samanta, C. Qin, A. J. Lough, G. A. Woolley, Angew. Chem. 2012, 124, 6558-656; (b) W. Moormann, D. Langbehn, R. Herges, Beilstein J. Org. Chem. 2019, 15, 727-732.

22. A. A. John, Q. Lin, J. Org. Chem. 2017, 82, 9873-9876.

23. C. Lucas-Lopez, N. Murphy, X. Zhu, Eur. J. Org. Chem. 2008, 4401-4404.

24. X.-M. Liu, X.-Y. Jin, Z.-X. Zhang, J. Wang, F.-Q. Bai, RSC Adv. 2018, 8, $11580-11588$.

25. J. García-Amorós and D. Velasco, Beilstein J. Org. Chem., 2012, 8, 1003-1017.

26. V. Chandrasekaran, E. Johannes, H. Kobarg, F. D. Sönnichsen, T. K. Lindhorst, ChemistryOpen 2014, 3, 99-108.

27. D. Wang, F. Schellenberger, J. T. Pham, H.-J. Butt, S. Wu, Chem. Commun. 2018, 54, 3403-3406.

28. A.-L. Leistner, S. Kirchner, J. Karcher, T. Bantle, M. L. Schulte, P. Gödtel, C. Fengler, Z. L. Pianowski, Chem. Eur. J. 2021, 27, 8094-8099.

29. M. W. Haydell, M. Centola, V. Adam, J. Valero, M. Famulok, J. Am. Chem. Soc. 2018, 140, 16868-16872.

30. F. Zhao, L. Grubert, S. Hecht, D. Bléger, Chem. Commun. 2017, 53, 3323-3326. 